\title{
Letter to the Editor: Hydrophobic interactions in the caseins: Challenging their dismissal by Holt et al. (2013)
}

\author{
David Horne ${ }^{1}$ and John A. Lucey \\ Department of Food Science, University of Wisconsin-Madison, Madison 53706
}

We are concerned about various assertions regarding the nature of casein interactions made in the recent review by Holt et al. (2013). Now, because this article is being cited by other researchers, we thought it would be helpful to raise these concerns to avoid misleading others. Previously (De Kruif and Holt, 2003), Holt tacitly accepted the notion of a dual-binding model (i.e., involving both attractive interactions and the formation of calcium phosphate nanoclusters as essential mechanisms for the assembly of casein micelles). But, in spite of the strong evidence in favor of their occurrence (e.g., dissociation by SDS or urea, temperaturedependent release of $\beta$-casein from micelles), Holt et al. (2013) now claim that the attractive components in casein micelle assembly are not hydrophobic interactions. They do this through a series of 3 statements. As we show below, the first statement is erroneous and also irrelevant. The second is correct but incomplete. The third misses the point of the mechanism it challenges.

First, Holt et al. (2013) claim we must reconsider the view that caseins are among the most hydrophobic proteins known to science. This must have come as a surprise to Fox and Brodkorb (2008), one of their supportive citations, for they noted only that $\beta$-casein was the most hydrophobic of the caseins. Holt et al. (2013) also cite Bigelow (1967) in support of their assertion, but closer inspection of the Bigelow data places $\alpha$-casein as little more than the average from his table of protein average hydrophobicities per residue. In reality, it matters little where the caseins rank in the spectrum of average hydrophobicities per residue. What matters for the development of hydrophobic interactions between these caseins is that their sequences possess clusters, or strings, of consecutive hydrophobic residues, as explained in the following.

For a hydrophobic solute to exist in an aqueous environment, a cavity must be created for it, disrupting the hydrogen bond structure between the water molecules. For small solutes, the free energy penalty so incurred increases with volume; however, for larger

Received January 30, 2017.

Accepted March 26, 2017.

${ }^{1}$ Corresponding author: brendavi@aol.com solutes, such as clusters of hydrophobic side chains, it shifts to a dependence on the surface area of the cavity created. Bringing together 2 hydrophobic clusters of amino acids is thus energetically favorable (attractive) because the surface of the cavity accommodating the combination is reduced by the sum of the areas of their original opposing interfaces. Moreover, the now close proximity of the opposing side-chains allows a further attractive component to come into play in the form of short-range Van der Waals and other dispersion forces. Hydrophobic interactions are now recognized in the modern chemistry field as a complex concerted process involving modification or rearrangement of aqueous hydrogen bonds as well as dispersion forces between hydrophobic side-chains.

Hydrophobic interactions are weak and therefore transient; however, it is their multiplicity and promiscuity that is so important in maintaining their aggregates or networks. Holt et al. (2013) make much of the example of the displacement of $\beta$-casein by denatured $\beta$-lactoglobulin. This result lies not so much in the relative strengths of individual hydrophobic interactions in these proteins but in the lateral disulfide cross-links, of which only $\beta$-lactoglobulin is capable, creating a $\beta$-lactoglobulin carpet, which is consequently more difficult to dislodge.

Holt et al. (2013) also suggest that likening $\beta$-casein self-association to the formation of detergent micelles is a poor example because no compact and anhydrous domain results, but this misses the fundamental point of the analogy. The 2 structures, $\beta$-casein micelles and detergent micelles, are the operational outcome of the same physics. The participating molecules each have a charged head (repulsive) and an attractive tail. Their aggregates are in equilibrium and organize themselves so that their repulsive centers are as far away from each other as the attraction of their tails allows. The tails therefore form a central core to the micelle and the charged heads an outer halo. The core density, or packing, is a function of both the repulsive (mainly) and attractive components. For the detergent micelle, with singly charged head groups, the repulsive element is low, whereas the attraction is high, because the hydrophobic interaction of the identical hydrocarbon tails is comple- 
mented by their short-range attractive Van der Waals forces. The result is close packing. For the $\beta$-casein micelle, the head groups with their phosphoserine clusters are heavily charged and distribute themselves across the surface of the micelle to minimize that repulsion, but are constrained from flying off into solution because of the attractive energy between hydrophobic segments in the tails making up the core. That this attraction is due to hydrophobic interactions is demonstrated when the hydrophobic clusters in the $\mathrm{C}$-terminal tail of $\beta$-casein are removed (Qi et al., 2005). The charge centers still provide a resultant radial pull outward from the center of the $\beta$-casein micelle, keeping the network from collapsing and, because there is no vacuum in solution, filling the interstices with water. This delicate balance of attractive and repulsive forces is the reason why the $\beta$-casein micelles are so highly solvated.

There are no substantive arguments for the dismissal of hydrophobic interactions between the caseins. You cannot airbrush out the discrete clusters of non-polar residues that the caseins contain. You cannot negate by diktat the ability of these clusters of non-polar residues to engage in hydrophobic interactions as and when the opportunity arises, as it inevitably will in their solutions. Although the precise molecular origin of hydrophobic interactions is still being debated, the effect of these interactions in casein systems has been well recognized for many decades. There are several other issues of concern in this review but these, and developments from them in later reviews from the Holt group, are discussed in a comprehensive review of casein interactions (Horne, 2017), which extends the arguments above.

\section{REFERENCES}

Bigelow, C. C. 1967. On the average hydrophobicity of proteins and relation between it and protein structure. J. Theor. Biol. 16:187-211.

De Kruif, C. G., and C. Holt. 2003. Casein micelle structure, functions and interactions. Pages 233-276 in Advanced Dairy Chemistry. Vol. 1A: Proteins: Basic Aspects. 3rd ed. P. F. Fox and P. L. H. McSweeney, ed. Kluwer, New York, NY.

Fox, P. F., and A. Brodkorb. 2008. The casein micelle: Historical aspects, current concepts and significance. Int. Dairy J. 18:677-684.

Holt, C., J. A. Carver, H. Eckroyd, and D. C. Thorn. 2013. Invited review: Caseins and the casein micelle: Their biological functions, structures, and behavior in foods. J. Dairy Sci. 96:6127-6146.

Horne, D. S. 2017. A balanced view of casein interactions. Curr. Opin. Colloid Interface Sci. 28:74-86. https://doi.org/10.1016/j. cocis.2017.03.009.

Qi, P. X., E. D. Wickham, E. G. Piotrowski, C. K. Fagerquist, and H. M. Farrell. 2005. Implications of C-terminal deletion on the structure and stability of bovine $\beta$-casein. Protein J. 24:431-444. 